\title{
Sistematização do conhecimento sobre as metodologias empregadas para o dimensionamento da força de trabalho em saúde
}

\author{
Systematization of the knowledge on methodologies for health \\ workforce staffing
}

Claudia Regina Machado', Mario Roberto Dal Poz²

\begin{abstract}
RESUMO A força de trabalho em saúde representa um fator de grande importância nos sistemas de saúde, sendo imprescindível à promoção e ao avanço da área, relacionada quantitativa e qualitativamente aos resultados alcançados. Esta revisão sistemática visa à identificação na literatura de metodologias que, empregadas, resultem em dimensionamento eficaz da força de trabalho em saúde. Os resultados indicam a necessidade de complementação da avaliação, sendo indispensável a identificação de metodologias que resultem em dimensionamento mais eficaz da força de trabalho em saúde para subsidiar decisões gerenciais no sistema de saúde.
\end{abstract}

PALAVRAS-CHAVE Dimensionamento; Força de trabalho; Carga de trabalho; Gestão de recursos humanos em saúde.

\begin{abstract}
The health workforce is a key factor in health systems and is essential to the promotion and advancement of the area, related quantitative and qualitative results achieved. This systematic review aims to identify, in the literature, methodologies that result in effective health workforce staffing. The results indicate the need to complement the assessment, in order to identify methodologies that result in more effective health workforce staffing and provide support to management decisions in the health system.
\end{abstract}

KEYWORDS Staffing; Labor force; Workload; Health personnel management.

1 Universidade do Estado do Rio de Janeiro (Uerj), Faculdade de Ciências Médicas, Departamento de Cirurgia Geral - Rio de Janeiro (RJ), Brasil. machado.c@globo.com 


\section{Introdução}

O Relatório da Organização Mundial da Saúde (2007) sobre os recursos humanos em saúde deixa clara a necessidade de vontade política, bem como da cooperação internacional, para o desenvolvimento e a implantação de planos nacionais direcionados e alinhados com a utilização de conhecimentos na construção e no reforço dos sistemas de saúde que contemplem tratamento e prevenção de doenças, além de promoção de saúde. Para tal, é imperioso o desenvolvimento de trabalhadores de saúde capazes e motivados para superar as dificuldades e alcançar os objetivos de desenvolvimento nacionais e globais.

Nos sistemas de saúde, a força de trabalho representa um fator de grande importância, sendo imprescindível à promoção e ao avanço da saúde, estando ligada quantitativa e qualitativamente aos bons resultados alcançados (OMS, 2007).

Estudos estatísticos prospectivos e progressivos demonstram que as doenças musculoesqueléticas decorrentes do aumento da expectativa de vida da população e da violência vêm crescendo progressivamente. O envelhecimento da população tende a resultar em aumento da utilização dos serviços hospitalares, decorrente de agravos característicos da terceira idade, neste caso particular em doenças reumatológicas e osteoporose (BRASIL, 2006, 2008; FARIZOTE, 2012). Além disso, morbimortalidade relacionada à violência e a acidentes de trânsito figura entre as principais causas de morte e de internação nos últimos anos no Brasil, apontando o caminho para a base das perspectivas de ampliação da capacidade instalada para atender às necessidades de atenção à saúde da população (BRASIL, 2006, 2008; FARIZOTE, 2012).

Nesse contexto, os gestores de saúde enfrentam desafios crescentes e recursos insuficientes em todo o mundo para responder à demanda de serviços de saúde. No Brasil, os gestores do Sistema Único de Saúde (SUS) têm procurado soluções para o desequilíbrio da força de trabalho, seja dentro das instituições, seja entre serviços de saúde (BRASIL, 2006).

O sistema de saúde brasileiro tem imensos desafios no que tange às demandas por serviços com qualidade, eficácia e eficiência, tornando fundamental a busca por melhor gerenciamento de recursos disponíveis (GAIDZINSKI, 2011). Deve ser ressaltado que a força de trabalho em saúde constitui um dos principais determinantes de eficiência e eficácia na prestação de cuidados em saúde.

Tradicionalmente, os países abordam as questões de planificação e de gestão de recursos humanos nos serviços de saúde com a utilização de desenhos baseados unicamente em razões de profissionais por população (ex.: médicos/habitante). Tal abordagem, embora ainda seja utilizada largamente para o cálculo de equipes e de profissionais, mostra-se insuficiente para adequar as necessidades de pessoal às diferenças epidemiológicas e sociais, bem como as necessidades dos serviços de saúde em matéria de efetivos e de formação.

Em outra abordagem, estipula-se a quantidade de atenção a ser oferecida por meio da normatividade da constituição de efetivos por estabelecimento, recorrendo-se à constituição de equipes padrão para o cálculo de lotação de pessoal para as diversas tipologias de serviços (postos e saúde, centros de saúde, hospitais etc.) e propondo-se uma equação entre capacidade instalada e quantitativo de pessoal. Ocorre que as necessidades de pessoal variam amplamente entre serviços de mesmo nível, de acordo com o volume do trabalho realizado (RAMOS; CRUZ; POLLARA, 2012).

Estudos já realizados se referem principalmente a metodologias de dimensionamento e avaliação de carga de trabalho de enfermagem por intermédio de avaliação das características da unidade analisada, média de permanência, taxa de ocupação, modelo de assistência, tipo de clientela, classificação de cuidados, composição da equipe, horas de trabalho e absenteísmo (HURST ET AL., 2008). 
Todavia, esses estudos, em sua maioria, indicam a necessidade de complementação para avaliação adequada, o que justificaria a necessidade de identificação de metodologias na literatura que possam ser empregadas em dimensionamento eficaz da força de trabalho em saúde, com a finalidade de fornecer subsídios às decisões gerenciais.

No Brasil, a metodologia de índice de carga de trabalho para necessidade de pessoal de saúde (Workload Indicators for Staffing Need - WISN) foi testada em uma unidade de saúde da família no município de Juiz de Fora (MG) e demonstrou sua utilidade para dimensionamento de médicos neste estabelecimento. Em relação à economicidade e à eficiência, tem potencial para mostrar caminhos para equacionar melhor distribuição de profissionais na rede de unidades de saúde, uma vez que aponta para sobrecarga ou ociosidade (PIERANTONI ET AL., 2011A, 2012B).

A escassez de modelos e metodologias para dimensionamento da força de trabalho para atuação em serviços de saúde que compreendam variáveis locais dificulta a gestão de recursos humanos no que tange à contratação, à alocação e à distribuição eficaz desses profissionais. A presente revisão da bibliografia sobre o dimensionamento de Recursos Humanos em Saúde (RHS) busca reduzir essa lacuna, bem como contribuir para o desenho e a utilização de modelos de organização e prestação de serviços que assegurem a oferta de serviços de saúde com qualidade e segurança.

\section{Metodologia}

Esta pesquisa foi elaborada por meio da estratégia de busca em bases de dados da literatura científica, filtragem de publicações, recuperação/aquisição de publicações científicas e análise descritiva preliminar do material recuperado com inclusão de fluxograma. Dessa forma, foi realizada revisão sistematizada da literatura científica sem corte temporal definido para identificar metodologias aplicadas para o dimensionamento dos profissionais da área da saúde.

A amostra foi constituída por estudos que preencheram os critérios de inclusão, selecionados pela pertinência ao tema e não por métodos aleatórios. Foram considerados os estudos não experimentais, descritivos, exploratórios e de abordagem qualitativa e quantitativa que tiveram como tema central metodologias para o dimensionamento da força de trabalho em saúde. Incluíram-se publicações nas línguas portuguesa, inglesa e espanhola.

Foram excluídos da pesquisa editoriais, cartas ao editor, erratas, artigos de opinião, documentos e resumos não encontrados na íntegra ou cuja aquisição era possível apenas por pagamento, e ainda documentos que não respeitaram os critérios de inclusão. Habilitaram-se todos os trabalhos que tiveram como escopo o uso de metodologias para o dimensionamento da força de trabalho em saúde ou de variáveis utilizadas para tal dimensionamento.

As bases de dados eletrônicas de literatura científica utilizadas foram: Embase, Medline, Lilacs, Wholis e o banco eletrônico de teses e dissertações. $\mathrm{O}$ acesso às bases de dados eletrônicas se deu mediante senha institucional ao Portal da Coordenação de Aperfeiçoamento de Pessoal de Nível Superior (Capes), acessado remotamente por identificação de endereço IP de provedor autorizado do Instituto de Medicina Social da Universidade do Estado do Rio de Janeiro. Seguem informações sobre as bases acessadas.

- Embase - É um serviço eletrônico da editora Elsevier que hospeda mais de 18 milhões de publicações contidas e acessadas na base europeia Embase isoladamente ou associada à base americana Medline. É a versão eletrônica das 52 seções da 'Excerpta Médica'. Contém mais de 15 milhões de registros bibliográficos com resumos da Embase (desde 1974) e Medline (desde 1966). 
- Lilacs - Literatura Latino-Americana e do Caribe em Ciências da Saúde. Trata-se de uma base de dados cooperativa do Sistema Bireme. Contém artigos de cerca de 670 revistas mais conceituadas da área da saúde, atingindo mais de 150 mil registros.

- Wholis - A biblioteca da Organização Mundial da Saúde (OMS) é a biblioteca líder mundial em saúde pública. Ele fornece acesso ao conteúdo da OMS, bem como a outras fontes de literatura científica produzida em todo o mundo. A Wholis também fornece evidências científicas e conhecimento para países de baixa e média rendas, por meio de um conjunto de iniciativas baixo custo/alto uso. Redes e parcerias são um componente essencial para garantir que suas iniciativas globais possam atingir um público mundial.

- Medline - É o componente primário do Pubmed. Abrange uma série de bases de dados providos pela biblioteca norte-americana de medicina (National Library of Medicine - NLM). O Pubmed é um serviço que inclui mais de 18 milhões de citações de artigos anteriores a 1950 e atualização semanal. Além das citações do Medline, traz links de diversos sites que acessam artigos em versão integral e outros recursos.

- Biblioteca Digital de Teses e Dissertações da Universidade de São Paulo (USP) - Foi criada para disponibilizar na Internet o conhecimento produzido pelos trabalhos defendidos na USP, permitindo que as comunidades brasileira e internacional possam ter em mãos a versão digital completa das teses e dissertações. A Biblioteca Digital está associada a uma iniciativa global reconhecida pela Organização das Nações Unidas para a Educação, a Ciência e a Cultura (Unesco), a Network Digital Library of Theses and Dissertations (NDLTD), o que lhe garante maior confiabilidade e abrangência, e também ao Instituto Brasileiro de Informação em Ciência e Tecnologia (IBICT) do Ministério da Ciência, Tecnologia e Inovação, por meio da Biblioteca Digital Brasileira de Teses e Dissertações.

$\mathrm{O}$ acesso às bases ocorreu durante junho de 2013, sendo complementado posteriormente em julho de 2014. Elegeram-se descritores a partir do vocabulário estruturado e trilíngue Descritores em Ciências da Saúde (DeCS), edição 2013, criado pelo Centro Latino-Americano e do Caribe de Informação em Ciências da Saúde (Bireme) e utilizado para buscas nas fontes de informação disponíveis na Biblioteca Virtual em Saúde (BVS).

\section{Resultados e discussão}

Os somatórios da busca de publicações nas bases eletrônicas estão apresentados na tabela 1.

Tabela 1. Resultado da busca eletrônica em bases de literatura científica. Brasil, 2014

\begin{tabular}{lrr}
\hline Bases & N. & \% \\
\hline Pubmed/Medline & 1.288 & 71,6 \\
Embase & 299 & 16,6 \\
Lilacs & 103 & 5,7 \\
BDTD & 48 & 2,7 \\
Banco USP & 39 & 2,2 \\
Wholis & 22 & 1,2 \\
Total & 1.799 & 100,00 \\
\hline
\end{tabular}

Fonte: Elaboração própria 
A observação da tabela 1 permite verificar que, a partir dos termos de busca utilizados sem os refinamentos ou descartes, as bases Pubmed/Medline e Embase foram aquelas que mais retornaram publicações: $71,6 \%$ e $16,6 \%$ do total, respectivamente.

Foi encontrado um somatório de 1.799 publicações nas diferentes bases de dados pesquisadas, do qual se descartaram 1.678, correspondentes a $93,3 \%$ dos estudos retornados.

A maior frequência de exclusão decorreu do fato de que as publicações abordavam outros temas $(87,2 \%)$, tais como saúde do trabalhador, rotatividade e fixação profissional sem utilização desses fatores para cálculos de dimensionamento profissional, e houve publicações repetidas (7,3\%). Em se tratando de publicações com idiomas diferentes de português, inglês ou espanhol, foram excluídos 51 estudos (3,0\%). Outros estudos $(n=25$; $1,5 \%)$ foram excluídos por se tratarem de artigos de opinião, editoriais, cartas ao editor e erratas, e por não terem sido encontrados ou cuja aquisição é mediante pagamento $(\mathrm{n}=17 ; 1,0 \%)$.

A figura 1 representa o fluxo dos resultados parciais de estudos elegíveis contidos nas bases de literatura científica, assim como os excluídos.

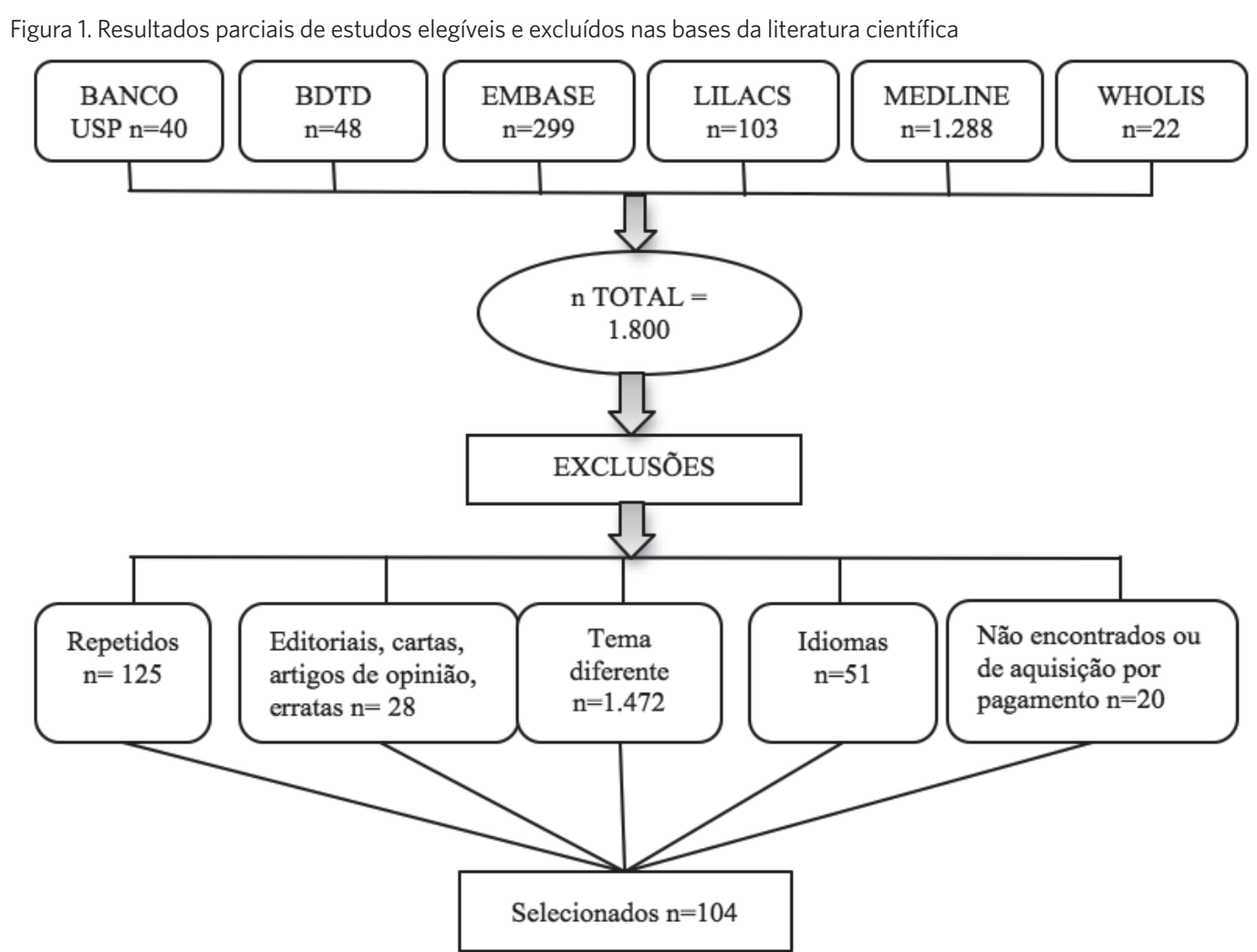

Fonte: Elaboração própria

No que concerne aos profissionais estudados, a maior parte foi de profissionais de enfermagem $(78,6 \%)$ de diferentes unidades. Médicos constituíram 8,7\% das pesquisas, as quais analisaram anestesiologistas, clínicos, cirurgiões, psiquiatras e patologistas. Alguns estudos abrangem pesquisas de mais de uma categoria profissional (3,9\%). Ressalta-se aqui que os estudos brasileiros de forma geral, com apenas uma exceção, referiam-se aos profissionais de enfermagem. 
As publicações selecionadas compreenderam o período de 1993 a 2013, tendo o maior número de estudos publicados entre os anos de 2008 e 2010. No que tange ao idioma, a língua inglesa predomina; há apenas um artigo em espanhol.

No que se refere aos artigos da categoria profissional enfermagem, alguns avaliam a estratificação da intensidade do cuidado objetivando melhor distribuição da equipe; muitos analisam a carga de trabalho, utilizando o Nurse Activities Score (NAS) ou resoluções do Conselho Federal de Enfermagem (Cofen); outros propõem a reorganização dos processos de trabalho e relocação da força de trabalho existente como solução para superar dificuldades no atendimento. Algumas publicações sugerem a utilização de instrumento de dimensionamento baseado na Classificação das Intervenções de Enfermagem (NIC), e um estudo valida esse instrumento para amostragem de carga de trabalho. O termo Classificação das Intervenções de Enfermagem compreende "o ordenamento ou arranjo das atividades de enfermagem dentro de um grupo ou dispostas numa base de relações e a determinação dos níveis de intervenções para estes grupos", enquanto a Taxonomia das Intervenções de Enfermagem significa "a organização sistemática dos níveis de intervenção baseada em semelhanças dentro da qual pode ser considerada uma estrutura conceitual" (MCCLOSKEY; BULECHEK, 1996 APUD GUIMARÃES; BARROS, 2001, P. 131).

Importante destacar que um estudo observacional prospectivo avalia a carga de trabalho da enfermagem pela utilização dos padrões da Associação Britânica de Medicina Perinatal. Outros dois artigos utilizaram a Resolução do Conselho Federal de Enfermagem (Cofen) no. 293/04 como parâmetro para dimensionamento da enfermagem. O artigo 11 desta resolução aponta dentre as competências privativas do enfermeiro: prescrição da assistência de enfermagem; cuidados diretos de enfermagem a pacientes graves com risco de morte; cuidados de enfermagem de maior complexidade técnica e que exijam conhecimentos de base científica e capacidade de tomar decisões imediatas. Além disso, nos artigos 12 e 13 encontramos as atividades exercidas pelo técnico e pelo auxiliar de enfermagem. $\mathrm{O}$ artigo 15 destaca que as atividades referidas nos artigos 12 e 13 da lei, quando exercidas em instituições de saúde, públicas e privadas, em programas de saúde, somente podem ser desempenhadas sob orientação, supervisão e direção do enfermeiro. A Resolução Cofen nº. 293/2004 fixa e estabelece parâmetros para o dimensionamento do quadro de profissionais de enfermagem nas unidades assistenciais das instituições de saúde e assemelhados. Entre os fatores importantes para cálculo de profissionais de enfermagem estão o modelo gerencial e assistencial; jornada de trabalho; dinâmica de funcionamento das unidades; índice de segurança técnica; taxa de absenteísmo; taxa de ausência de benefícios e Sistema de Classificação de Pacientes (SCP) (MARINHO, 2009). Esses artigos estão listados no quadro 1.

\begin{tabular}{lllll}
\hline \multicolumn{2}{l}{ Quadro 1. Artigos selecionados sobre a categoria profissional de enfermagem } & & \\
\hline ABORDAGEM & FOCO & ABRANGÊNCIA & PERIÓDICO & AUTOR E ANO \\
\hline $\begin{array}{l}\text { Avaliação de ferramenta de carga de trabalho } \\
\text { para unidade neonatal }\end{array}$ & Hospital & Local & J. Nurs. Manag. & SAWATZKY-DICKSON; \\
$\begin{array}{l}\text { Capacidade instalada, tempo de execução de } \\
\text { atividades, carga horária }\end{array}$ & Hospital & Local & Rev. Bras. de Cancerologia & ALMEIDA; JUNQUEIRA; \\
\begin{tabular}{l} 
Carga de trabalho e comunicação \\
\hline
\end{tabular} & Ambulatório & Local & Nurs. Econ. & DICKSON et al., 2010 \\
\hline
\end{tabular}


Quadro 1. Artigos selecionados sobre a categoria profissional de enfermagem

\begin{tabular}{|c|c|c|c|c|}
\hline Carga de trabalho por grupo de profissionais & Hospital & Local & Nurs. Forum & WILLIS et al., 2012 \\
\hline Carga de trabalho/hora ou dia $\times$ Custo & Hospital & Local & Nurs. Manage. & STENSKE; FERGUSON, 1996 \\
\hline Classificação de intervenção & Hospital & Local & Rev. Esc. Enf. USP & GUIMARÃES; BARROS, 2001 \\
\hline Cofen, 2004 & Hospital & Local & Rev. Admin. Saúde & COLETTA; PROCHET, 2005 \\
\hline Cofen, 2004 & Hospital & Local & Rev. Bras. de Terapia Intensiva & GARCIA, P. C., 2011 \\
\hline Cofen, 2004 & Hospital & Local & $\begin{array}{l}\text { Rev. Latino-Americana de } \\
\text { Enfermagem }\end{array}$ & ARAÚJO, V. B. et al., 2009 \\
\hline Cofen, 2004 & Hospital & Local & Tese (Doutorado) -, USP & NICOLA, 2004 \\
\hline Cofen, 2004 & Hospital & Nacional & Tese (Livre Docencia) -, USP & FUGULIN, 2010 \\
\hline $\begin{array}{l}\text { Comparação de classificação de risco com } \\
\text { outros métodos de dimensionamento }\end{array}$ & Hospital & Local & Nurs. Manage. & ADAMS, L. W., 2003 \\
\hline Custos x Produção e capacidade operacional & Hospital & Local & Rev. Admin. Saúde & RAMOS; CRUZ; POLLARA, 2012 \\
\hline $\begin{array}{l}\text { Desenvolvimento de método computadorizado } \\
\text { qualidade- necessidade } \mathrm{RH}\end{array}$ & Hospital & Local & Burns & $\begin{array}{l}\text { JONG; LEEMAN; MIDDEL } \\
\text { KOOP, } 2009\end{array}$ \\
\hline Determinação de carga de trabalho & Hospital & Local & $\begin{array}{l}\text { International Journal of } \\
\text { Nursing Studies }\end{array}$ & TWIGG; DUFFIELD, 2009 \\
\hline $\begin{array}{l}\text { Média de permanência e características da } \\
\text { unidade por meio de entrevistas }\end{array}$ & Hospital & Nacional & Cogitare Enferm. & CAMPOS; MELO, 2009 \\
\hline Estudo observacional & Hospital & Local & J. Adv. Nurs. & ADOMAT; HICKS, 2003 \\
\hline Estudo observacional prospectivo & Hospital & Local & S. Afr. Med. J. & WALLIS; TWOMEY, 2007 \\
\hline Estudo retrospectivo baseado em opiniões & Hospital & Local & J. Nurs. Manag. & RABY; DWSE, 2008 \\
\hline Cofen & Hospital & Local & Acta Paul. Enferm. & $\begin{array}{l}\text { MATSUSHITA; ADAMI; } \\
\text { CARMAGNI, } 2005\end{array}$ \\
\hline $\begin{array}{l}\text { Grau de dependência e necessidade de saúde } \\
\text { da população }\end{array}$ & Hospital & Local & Br. J. Community Nurs. & REID; KANE; CURRAN, 2008 \\
\hline $\begin{array}{l}\text { Horas de assistência/categoria profissional e } \\
\text { capacidade cirúrgica }\end{array}$ & Hospital & Local & Dissertação (Mestrado) -USP & MATTIA, 1998 \\
\hline Indicadores & Hospital & Local & Tese (Doutorado), USP & POSSARI, 2011 \\
\hline Indicadores & Hospital & Local & Dissertação (Mestrado), USP & KROKOSCZ, 2007 \\
\hline Indicadores de tempo & Hospital & Local & Tese (Doutorado), USP & MELLO, 2011 \\
\hline Medida da distribuição da carga de trabalho & Hospital & Local & Ann. Emerg. Med. & LEVIN et al., 2007 \\
\hline Modelo computadorizado & Hospital & Local & J. Nurs. Manag. & GHOSH B, CRUZ G, 2005 \\
\hline $\begin{array}{l}\text { Modelo de tempo de cuidado - grau de } \\
\text { dependência }\end{array}$ & Hospital & Local & J. Med. Syst. & SOLIMAN, 1997 \\
\hline
\end{tabular}

Fonte: Elaboração própria

*NEM - Nine Equivalents of Nursing Manager Use Score

${ }^{\star *}$ Coeficiente de Pearson - medida de correlação linear entre duas variáveis

O NAS é baseado no desenvolvimento de índices de gravidade de pacientes, o Therapeutic Intervention Scoring System (Tiss), que após várias versões foi reestruturado e mais direcionado para avaliar as necessidades de cuidados e carga de trabalho de enfermagem. Passou a se denominar Nursing Activities Score (NAS), incorporando atividades de enfermagem não contempladas nas versões anteriores, como procedimentos de higiene, suporte e cuidados aos familiares/pacientes, tarefas administrativas e gerenciais. O escore $N A S$ expressa a porcentagem de tempo gasto por um profissional de enfermagem na assistência direta ao doente crítico na UTI em 24 horas (GONÇALVES, 2006). Os artigos estão agrupados no quadro 2. 
Quadro 2. Artigos selecionados sobre a categoria profissional de enfermagem, com abordagem de Nursing Active Score, foco em hospital e abrangência local

\begin{tabular}{ll}
\hline \multicolumn{1}{c}{ PERIÓDICO/FONTE } & \multicolumn{1}{c}{ AUTOR E ANO } \\
\hline Biblioteca Digital, UEL & CAMUCl et al. , 2012 \\
Biblioteca Digital, USP & TSUKAMOTO, 2010 \\
Biblioteca Digital, USP & GOUVEA, 2012 \\
Critical Care Medicine & MIRANDA et al., 1996 \\
Intensive Crit. Care Nurs. & PADILHA et al., 2007 \\
Biblioteca Digital, USP & GONÇALVES et al., 2006 \\
Acta Paulista de Enfermagem & DUCCI et al., 2007 \\
Biblioteca Digital, USP & QUEIJO, 2008 \\
Acta Paulista de Enfermagem & LIMA, 2010 \\
Biblioteca Digital, USP & KAKUSHI, 2012 \\
\hline
\end{tabular}

Fonte: Elaboração própria

O uso de tecnologia também foi avaliado tanto para o dimensionamento da enfermagem como para o auxílio às tarefas, visando diminuir assim a necessidade de pessoal por meio de realocação. Diers e Bozzo (1997) mostraram que novos softwares desenvolvidos para a saúde podem fornecer informações integradas do registro clínico dos pacientes, além de proporcionar maior controle nos gastos.

No que tange à alta complexidade, foram encontrados apenas três artigos relacionados ao câncer, sendo dois brasileiros e um italiano, descritos a seguir.

Almeida, Junqueira e Oliveira (2007) estudaram o estabelecimento de parâmetros adequados ao dimensionamento da força de trabalho necessária ao atendimento de ações realizadas por unidades hospitalares especializadas de alta complexidade de assistência ao câncer no Instituto Nacional de Câncer (Inca). A metodologia adotada relaciona a capacidade instalada, o tempo de execução das atividades, a carga horária e o quantitativo das categorias profissionais necessárias ao adequado atendimento das demandas. $\mathrm{O}$ estudo aponta como estratégico o correto dimensionamento das equipes de enfermagem para se obterem resultados de produtividade e qualidade, considerando as características de continuidade ininterrupta e diversidade das atividades desenvolvidas pelas categorias de enfermagem, além do seu expressivo peso no conjunto da força de trabalho da área de saúde. Como resultado, observou-se que o quantitativo necessário ao desenvolvimento do conjunto de atividades das cinco unidades estudadas representa $50 \%$ dos recursos humanos. Na área médica, a proporção de pessoal em relação ao total correspondeu a $20 \%$. Nas áreas de apoio, o percentual correspondente à necessidade de recursos humanos foi de $11 \%$. No Hospital do Câncer IV (HC IV), no entanto, em razão das atividades de visitas domiciliares, essas atividades têm um impacto de $26 \%$ em relação ao total da unidade.

Ramos, Cruz e Pollara (2012) estudaram o desenvolvimento e a aplicação de metodologia para dimensionamento do corpo clínico de um hospital de ensino no município de São Paulo, utilizando dados de produção e capacidade operacional - considerando o fato de ser uma instituição de ensino -, e fizeram correlação com os custos. Esse estudo concluiu que a simples realocação da carga horária, com melhor distribuição do tempo entre as especialidades e os tipos de atendimento, levaria à correção de distorções, resultando em economia do ponto de vista financeiro (quadro 3). 
Quadro 3. Artigos selecionados sobre a categoria profissional de médicos

\begin{tabular}{|c|c|c|c|c|}
\hline ABORDAGEM & FOCO & ABRANGÊNCIA & PERIÓDICO & AUTOR E ANO \\
\hline Comparação tempo de cuidado & Hospital & Local & Psychiatr. Serv. & BHASKARA, 1999 \\
\hline $\begin{array}{l}\text { Estudo prospectivo da gravidade } \\
\text { dos pacientes }\end{array}$ & Hospital & Local & S. Afr. Med. J. & $\begin{array}{l}\text { WALIS; TWOMEY, } \\
2007\end{array}$ \\
\hline $\begin{array}{l}\text { Modelo empírico para análise do } \\
\text { tempo de atividade }\end{array}$ & Hospital & Nacional & $\begin{array}{l}\text { Bull. World Health } \\
\text { Organ. }\end{array}$ & $\begin{array}{l}\text { DAVIAUD; CHOPRA, } \\
2008\end{array}$ \\
\hline $\begin{array}{l}\text { Custos x Produção e capacidade } \\
\text { operacional }\end{array}$ & Hospital & Local & $\begin{array}{l}\text { Rev. de Administração } \\
\text { em Saúde - RAS }\end{array}$ & $\begin{array}{l}\text { RAMOS; CRUZ; } \\
\text { POLLARA, } 2012\end{array}$ \\
\hline $\begin{array}{l}\text { Medida da distribuição da carga } \\
\text { de trabalho }\end{array}$ & Hospital & Local & Ann. Emerg. Med. & LEVIN et al., 2007 \\
\hline Modelo empírico & Hospital & Local & J. Oncol. Pract. & $\begin{array}{l}\text { FASOLA; APRILE; } \\
\text { AITA, } 2012\end{array}$ \\
\hline Modelo empírico & Hospital & Local & Health Serv. Res. & LIPSCOMB et al., 1995 \\
\hline Número de profissionais & Hospital & Nacional & Ann. Emerg. Med. & $\begin{array}{l}\text { MOORHEAD et al., } \\
2007\end{array}$ \\
\hline Questionário & Hospital & Local & Critical. Care Nurse. & HARTIGAN, 2000 \\
\hline Sistema Warwick & Hospital & Local & J. Clin. Pathol. & CARR et al., 2006 \\
\hline
\end{tabular}

Fonte: Elaboração própria

Fasola, Aprile e Aita (2012) realizaram estudo para estimar as necessidades de recursos humanos para o tratamento de pacientes com câncer em um hospital universitário italiano. A metodologia utilizada considerou o número e os diferentes tipos de atendimento, além de estimar custos de pessoal envolvido, correlacionando com a carga de trabalho. A conclusão do estudo foi a de que o modelo utilizado poderá ser útil a gestores e de que médicos oncologistas podem ter participação mais ativa nas políticas de saúde (quadro 3).

Em relação aos médicos, também foram encontrados três artigos referentes a serviços de emergência, baseados em estudos observacionais prospectivos, avaliando a gravidade dos casos atendidos (WALIS; TWOMEY, 2007) e os horários de pico de atendimento (BHASKARA, 1999). Foi selecionado ainda artigo referente à medida de carga de trabalho por meio de avaliação de informações contidas em sistemas de informação (LEVIN ET AL., 2007) (quadro 3).

O desenvolvimento de metodologias capazes de estabelecer parâmetros para alocação de profissionais que auxiliem os gestores a dimensionar o quadro de pessoal de forma racional - com adequação de custos condizentes com a realidade de escassez financeira e concomitantemente melhorando o serviço prestado ou programando novas modalidades de assistência - levou a Organização Mundial da Saúde a propor metodologia para cálculo de pessoal em 1998, denominada de Indicadores de Carga de Trabalho para Necessidade de Pessoal, em inglês, Workload Indicators of Staffing Need (WISN).

Foram encontradas publicações referentes à aplicação do método de indicadores de carga de trabalho em unidades de Atenção Básica e em países em desenvolvimento. Não foi encontrada nenhuma referência da utilização desse método para alta complexidade em ortopedia, assim como não há descrição referente ao uso da ferramenta de cálculo (software) após as atualizações e a tradução para a língua portuguesa. Essa metodologia foi utilizada na Tanzânia, no Quênia, em Moçambique, Sri Lanka, Sudão, Turquia e Egito e vem sendo aprimorada gradativamente (NAMAGANDA, 2010; NYANTEMA ET AL., 2008).

Na Tanzânia, os problemas de saúde de maior importância estão relacionados à escassez de profissionais qualificados, 
especialmente em relação à mortalidade e morbidade materna e perinatal. Diante desse quadro, foram projetados indicadores de carga de trabalho utilizando o método WISN, em 2004, para cálculo da necessidade de enfermagem, o que evidenciou enorme carga de trabalho e necessidade urgente de aumento dessa força de trabalho, para que haja possibilidade de atingir os objetivos globais de desenvolvimento do milênio (NYANTEMA ET AL., 2008).

Estudos de casos também foram realizados na Indonésia, em Moçambique e Uganda, utilizando o mesmo método para avaliar a necessidade de aumento da força de trabalho em saúde e propor recomendações baseadas em evidências e critérios técnicos. Estudaram-se diferentes classes profissionais nos três países e com abrangência também distinta entre eles. No entanto, verificou-se que quando o WISN é aplicado para um grupo de profissionais que trabalham em conjunto e não para uma única categoria profissional os resultados são mais valiosos, como no caso de Moçambique (SERAN ET AL., 2010).

Em Bangladesh, o estudo foi proposto para avaliar enfermeiros, médicos e assistentes médicos, mostrando baixa ocupação dos leitos disponíveis, em virtude da escassez desses profissionais. Em algumas localidades, só havia disponibilidade de serviços ambulatoriais (HOSSAIN; ALAM, 1999).

Em todos os estudos fica claro que o método WISN é uma ferramenta para orientação de alocação da força de trabalho em saúde em seus diferentes níveis de complexidade, que visa proporcionar distribuição mais equilibrada de seus recursos humanos como resultado da análise.

Os Indicadores de Carga de Trabalho (WISN) usam a análise de atividade com a carga de trabalho para determinar as necessidades de recursos humanos, podendo ser utilizados como ferramenta para planejamento e gestão, melhorando as decisões em todos os níveis de serviço, tanto no fornecimento quanto na alocação e na montagem de equipe.
Para entender o conceito de carga de trabalho é preciso compreender a forma de produção, a organização do trabalho e sua divisão, já que é um conceito que tenta estabelecer dinâmica entre o trabalhador e seu trabalho (PIERANTONI ET AL., 2011A, 2011B).

Para o método WISN, é central o cálculo da carga de trabalho padrão, que é igual ao tempo de trabalho disponível no ano dividido pela unidade de tempo. Vale ressaltar que todos os cálculos utilizados têm como base 12 meses e são retrospectivos. Os componentes da carga de trabalho são representados pelas atividades de serviço de saúde, de apoio e outras.

Dentre as limitações do método, talvez a de maior impacto seja referente a não se considerar a influência de fatores externos, como a escassez de hemoderivados nos casos cirúrgicos, além de a fonte de dados ser pobre e incorreta, o que poderá levar a resultados imprecisos e indicações equivocadas (HOSSAIN; ALAM, 1999).

Outra limitação é salientada por Pierantoni et al. (2011A, 2011B) em relação aos indicadores de carga de trabalho para profissionais: a de que o método está baseado nas cargas de trabalho atuais, ou seja, na produção de serviços do momento, não considerando a demanda não atendida ou reprimida. Em relação à Atenção Básica, apesar de o dimensionamento de pessoas referente à saúde ser pouco discutido (PIERANTONI ET AL., 2011A, 2011B), confirma-se que na literatura nacional há grande concentração de títulos ligados à enfermagem. No entanto, observa-se que os métodos utilizados não consideram importantes variações locais, como quadro epidemiológico, necessidades e contexto socioeconômico, taxas de morbimortalidade e acesso aos serviços de saúde.

\section{Conclusões}

Dos artigos selecionados, 22 (vinte e dois) deles, apesar de abordarem o tema de dimensionamento de pessoal, não 
apresentavam metodologia clara, sendo algumas subjetivas, outras apenas quantificando o número de profissionais ou planejamento para melhor aproveitamento da capacidade instalada.

Além disso, várias publicações - tanto do Ministério da Saúde quanto da Fundação Oswaldo Cruz (Fiocruz) e da Organização Pan-Americana da Saúde - revelam o envelhecimento da população associado ao aumento da morbimortalidade, ou seja, doenças associadas à terceira idade e causas externas como aumento da violência urbana, impulsionando a urgência de um dimensionamento da força de trabalho em saúde em todos os seus níveis de complexidade que possam ser eficientes e eficazes.

O Ministério da Saúde da Nicarágua (2007) publicou um manual que ressalta a importância da mudança na gestão de recursos humanos, considerando a necessidade de educação para intervenções como regulação, planejamento e gestão, entre outras, para oferecer equidade com qualidade, indicando para tal a necessidade de políticas e investimentos nessa área. A Organização Mundial da Saúde, desde 2006, vem mostrando a importância da adequada gestão dos recursos humanos em saúde para o alcance de resultados satisfatórios tanto para prevenção como para promoção à saúde, assim como para o tratamento.

Diante dos métodos utilizados, podemos observar que todos apresentam alguma limitação e que a maioria dos estudos é referente à enfermagem. As outras categorias profissionais são pouco representadas e estudadas. Fica claro que nenhuma metodologia de forma isolada dá conta de tantos processos envolvidos no dimensionamento, na distribuição e na alocação desses profissionais.

Outro ponto a ser destacado é o pequeno número de publicações referentes aos médicos, mesmo considerando que em algumas regiões há escassez desses profissionais. Ainda quando o quantitativo pareça adequado, nem todos estão capacitados para a realização dos procedimentos e técnicas necessários.

Os poucos estudos que contemplam todas as categorias da saúde envolvidas no cuidado são relativos à Atenção Básica. Em todos os estudos que utilizaram o método de Indicadores de Carga de Trabalho para Necessidade de Pessoal, fica claro que ele é uma ferramenta para orientação de alocação da força de trabalho em saúde em seus diferentes níveis de complexidade, que visa proporcionar distribuição mais equilibrada de seus recursos humanos como resultado da análise. No entanto, deve ser ressaltada a limitação na consideração aos fatores externos, como problemas estruturais e falta de material, entre outros, o que pode levar a análises imprecisas.

\section{Recomendações}

Com os resultados obtidos espera-se poder subsidiar, com sustentação metodológica, o dimensionamento dos profissionais de saúde na rede de serviços em todos os níveis, seu delineamento operacional e implantação, otimizando tanto a capacidade profissional como o desempenho dos profissionais.

A partir dessa avaliação é possível compartilhar o conhecimento sobre vantagens e problemas no uso de metodologia baseada na carga de trabalho, visando contribuir para o desenvolvimento de outras opções metodológicas e consequente melhoria na composição de equipes de profissionais de saúde qualificados, gerando assim maior qualidade de vida e saúde da população.

Vale ressaltar que todas as metodologias utilizadas têm como objetivo planejar as necessidades de pessoal nas unidades de saúde, sendo necessária na maioria dos casos a associação de mais de uma para a realização de projeções futuras dessas necessidades. Além disso, deve ser considerado o fato de que algumas análises podem gerar impacto em 
diferentes condições de emprego, incluindo mudanças de regime de trabalho, novos direitos ou incentivos ou ainda a introdução de novos programas de formação e capacitação.

Dessa forma, em consonância com os princípios de democratização do conhecimento, este estudo possibilita o acesso à produção e ao debate atual relevantes do campo da saúde coletiva, relacionados ao dimensionamento de pessoal da saúde no SUS e mesmo nos sistemas complementares no território nacional.

\section{Agradecimentos}

Os autores agradecem a Deise Brasil e a Maria Helena Costa Couto pelos comentários e valiosas sugestões durante a redação deste artigo.

\section{Referências}

ADAMS, L. W. Clocking care hours with workload measurement tools. Nursing Management, Philadelphia, v. 34, n. 8, p. 34-39, 2003.

ADAMS, L. W. et al. Budgeting nursing workload for required minimum data set assessments. Journal of Nursing Management, Oxford, v. 15, n. 4, p. 442-448, 2007.

ADOMAT, R.; HICKS, C. Measuring nursing workload in intensive care: an observational study using closed circuit video cameras. Journal of Advanced Nursing, Oxford, v. 42, n. 4, p. 402-12, 2003.

AIKEN, L. H. et al. Implications of the California nurse staffing mandate for other states. Health Service Research, Chicago, v. 45, n. 4, p. 904-921, 2010.

ALMEIDA, V. M. L.; JUNQUEIRA, A. R. A.; OLIVEIRA, E. S. Perfil da força de trabalho do Inca. Revista Brasileira de Cancerologia, Rio de Janeiro, v. 53, n. 1, p. 87-98, 2007.

ARAÚJO, V. B. et al. Variabilidade do grau de complexidade assistencial do paciente em relação à equipe de enfermagem. Revista Latino Americana de Enfermagem, Ribeirão Preto, v. 17, n. 1, p. 34-39, 2009.

BHASKARA, S. M. Setting benchmarks and determining psychiatric workloads in community mental health programs. Psychiatric Services Journal, Maryland, v. 50, n. 5, p. 695-697, 1999.

BOMFIM, D. Identificação das intervenções de enfermagem na Atenção Básica à Saúde como parâmetro para o dimensionamento de trabalhadores. Revista da Escola de Enfermagem da USP, São Paulo, v. 46, n. 6, p.1462-1470, 2010.

BRASIL. Ministério da Saúde. Portaria n.2.528 de 19 de outubro de 2006. Aprova a Política Nacional de Saúde da Pessoa Idosa. 2006. Disponível em: <http://www.saudeidoso.icict.fiocruz.br/pdf/ PoliticaNacionaldeSaudedaPessoaIdosa.pdf $>$. Acesso em: 10 jul. 2014.

Ministério da Saúde. Temática Prevenção de Violências e Cultura da Paz III. Painel de Indicadores do SUS n.5: Brasília, DF: Organização Pan-Americana da Saúde, 2008.

CAMPOS, L. F.; MELO, M. R. A. C. Dimensionamento de pessoal de enfermagem: parâmetros, facilidades e desafios. Cogitare Enfermagem (UFPR), Curitba, v. 14, n. 2, p. 237-246, 2009.

CAMUCI, M. B. Carga de trabalho de enfermagem em unidades de terapia intensiva de Queimados segundo o Nursing Activities Score. 2012. 108p. Dissertação (Mestrado em Enfermagem) - Universidade Estadual de Londrina, Londrina, 2012.

CARR, R. A. GRASPing the nettle, the introduction of a workload measurement tool into an accident and emergency department. Accid. Emerg. Nurs., Philadelphia, v. 2, n. 1, p. 21-26, 1994.

CARR, R. A. et al. The Warwick system of prospective workload allocation in cellular pathology - an aid to 
subspecialisation: a comparison with the Royal College of Pathologists' system. J Clin Pathol., Londres, v. 59, n. 8, p. 835-839. 2006.

COLLETA, M. M. D.; PROCHET, T. C. Comparação de diferentes parâmetros para dimensionamento da equipe de enfermagem em um hospital universitário. Rev. Adm. Saúde, São Paulo, v. 7, n. 26, p. 19-24, jan./mar. 2005.

CRUZ, C. W. M. Construção de instrumento de medida do tempo de trabalho da enfermagem em centro diagnóstico por imagem. 2012. 109 p. Dissertação (Mestrado em Enfermagem) - Escola de Enfermagem, Universidade de São Paulo, São Paulo, 2012.

DAL BEM, L. W. Dimensionamento do pessoal de enfermagem em assistência domiciliária: percepção de gerentes e enfermeiras. 2005. 169f. Tese (Doutorado em Enfermagem) - Escola de Enfermagem, Universidade de São Paulo, São Paulo, 2005.

DAVIAUD, E.; CHOPRA, M. How much is not enough? Human resources requirements for primary health care: a case study from South Africa. Bulletin of. World Health Organization, Genebra, v. 86, n. 1, p. 46-51, 2008.

DICKSON et al. Nursing workload measurement in ambulatory care. Nurs. Econ., New Jersey. v. 28, n. 1, p. $37-43,2010$.

DIERS, D.; BOZZO, J. Nursing resource definition in DRGs. RIMS/Nursing Acuity Project Group. Nursing Economics, Pitman, v. 15, n. 3, p.124-130, 1997.

DRAKE, R. Nursing workforce planning: insights from seven Malasyan Hospitals. Br. J. Nurs. Londres, v. 22, n. 2, p. 95-100, 2013.

DUCCI, A. J. et al. Nursing Activies Score (NSA): Estudo comparative dos resultados da aplicação retrospectiva e prospectiva em Unidade de Terapia Intensiva. Acta Paulista de Enfermagem, São Paulo, v. 21, n. 4, p. 581-587, 2007.

DUFFIELD, C.; ROCHE, M.; MERRICK, E. T. Methods of measuring nursing workload in Australia. Collegian, Subiaco, v.13, n.1, p.16-22, 2006.
FARIZOTE, B. Análise estratégica da modalidade hospital-dia na unidade hospitalar do Instituto Nacional de Traumatologia e Ortopedia. 2013. 146 f. Dissertação (Mestrado em Saúde Pública) - Fundação Oswaldo Cruz, Rio de Janeiro, 2013.

FASOLA, G.; APRILE, G.; AITA, M. A model to estimate human resource needs for the treatment of outpatients with cancer. J. Oncol. Pract., Alexandria, v. 8, n. 1, p. 13-17, 2012.

FUGULIN, F. M. T. Parâmetros Oficiais para o Dimensionamento de Profissionais de Enfermagem em Instituições Hospitalares: análise da Resolução COFEN No 293/04. 2010. 154 f. Tese (Livre docência) - Escola de Enfermagem, Universidade de São Paulo, São Paulo, 2010.

GAIDZINSKI, R. R. Dimensionamento informatizado de profissionais de enfermagem: desenvolvimento de um software. In: PIERANTONI, C. R.; DAL POZ, M. R.; FRANÇA, T. (Org.). O trabalho em saúde: abordagens quantitativas e qualitativas. 11 ed. Rio de Janeiro: CEPESC, 2011. v. 1, p. 91-100.

GONÇALVES, L. A. et al. Fatores associados à carga de trabalho de enfermagem em unidade de terapia intensiva de adultos no primeiro dia de internação. 2006. 82 f. Dissertação (Mestrado em Enfermagem) Universidade de São Paulo, São Paulo, 2006.

GHOSH, B.; CRUZ, G. Nurse requirement planning: a computer-based model. J. Nurs. Manag., Oxford, v. 13, n. 4, p. 363-367, 2005.

GOUVEA, P. B. Relação entre horas requeridas e horas disponíveis de assistência de enfermagem em Unidade de Terapia Intensiva. 2012. Dissertação (Mestrado em Enfermagem Fundamental) - Escola de Enfermagem de Ribeirão Preto, Universidade de São Paulo, Ribeirão Preto, 2012.

GUIMARÃES, H. C. Q. C. P.; BARROS, A. L. B. L. Classificação das intervenções de Enfermagem. Revista da Escola de Enfermagem da Universidade de São Paulo, São Paulo, v. 5, n. 2, p. 130-134, 2001.

HARTIGAN, R. C. The synergy model establishing 
criteria 1:1 staffing ratios. Critical care nurse, Boston, v. 20, n. 2, p. 112, 114-16. 2000.

HOSSAIN, B.; ALAM, S. A. Workload indicators staffing need for human resource management and planning in the health sector of Bangladesh. Human Resources for Health Development Journal (HRDJ), Bangkok, v. 3, n. 2, p. 1-14, 1999.

HURST, K. et al. Calculating staffing requirements. Nursing Management (Harrow), Philadelphia, v. 15, n. 4, p. 26-34, 2008.

JEANG, A. Flexible nursing staff planning with adjustable patient demands. Journal of Medical Systems, New York, v. 20, n. 4, p. 173-182, 1996.

JONG A, E.; LEEMAN, J.; MIDDEL KOOP, E. Development of a nursing workload measurement instrument in burn care. Burns, Guildford, v. 35, n. 7, p. 942-948, 2009.

KAKUSHI, L. E. Mensuração e análise do tempo de assistência de enfermagem direta e indireta em unidade de terapia intensiva. 2012. 83f. Dissertação (Mestrado em Ciências) - Escola de Enfermagem de Ribeirão Preto, Universidade de São Paulo, Ribeirão Preto, 2012.

KROKOSCZ, D. V. C. Efeitos da alocação de pessoal e de carga de trabalho de enfermagem nos resultados da assistência em unidades de internação médico-cirúrgicas. 2007. 103f. Dissertação (Mestrado em Enfermagem na Saúde do Adulto) - Escola de Enfermagem, Universidade de São Paulo, São Paulo, 2007.

LEVIN, S. et al. Shifting toward balance: measuring the distribution of workload among emergency physician teams. Ann. Emerg. Med., Lansing, v. 50, n. 4, p. 419-423, 2007.

LIPSCOMB, J. et al. Determining VA physician requirements through empirically based models. Health Serv Res, Chicago, v. 29, n. 6, p. 697-717, 1995.

LYNEHAM, J.; CLOUGHESSY, L.; MARTIN, V.

Workloads in Australia emergency departments a descriptive study. Int Emerg Nurs, Oxford, v. 16, n. 3, p. 200-206. 2008.
MAGALHÃES, A. M. Carga de trabalho da equipe de enfermagem e segurança do paciente: estudo com método misto na abordagem ecológica restaurativa. Revista Latino-Americana de Enfermagem, Ribeirão Preto, Universidade de São Paulo, v. 21, n. spe, p.1-9, fev. 2013.

MARINHO, A. M. Dimensionamento de profissionais de enfermagem baseado na resolução Cofen 293/2004. Relatório Técnico COFEN. Rio de Janeiro: COFEN, 2009.

MATSUSHITA, M. S.; ADAMI, N. P.; CARMAGNI, M. I. Dimensionamento do pessoal de enfermagem das unidades de internação do Hospital São Paulo. Acta Paulista de Enfermagem, São Paulo, v. 18, n. 1, p. 9-19, 2005.

MATTIA, A. L. Dimensionamento de pessoal de enfermagem em centro cirúrgico. 1998. 108f. Dissertação (Mestrado em Enfermagem) - Escola de Enfermagem, Universidade de São Paulo, 1998.

MELLO, M. C. Carga de trabalho de enfermagem: indicadores de tempo em unidades de clínica médica, cirúrgica e terapia intensiva adulto. 2011. 228p. Tese (Doutorado em Ciências) - Escola de Enfermagem, Universidade de São Paulo, São Paulo, 2011.

MILLIGAN, D. W. et al. Nursing workload in UK tertiary neonatal units. Arch. Dis. Child, Londres, v. 93, n. 12, p. 1059-1064, 2008

MIRANDA, D. R. et al. Simplified Therapeutic Intervention Scoring System: the TISS-28 items--results from a multicenter study. Critical Care Med, Philadelphia, v. 24, n. 1, p. 64-73, 1996.

MOORHEAD, J. C. et al. A study of the workforce in emergency medicine. Ann. Emergency Medicine, Lansing, v. 27, n. 6, p. 691-700, 2007.

MYNY, D. et al. Determination of standard times of nursing activities based on a Nursing Minimum Dataset. Journal of Advanced Nursing, Oxford, v. 66, n. 1, p. 92-102, 2010.

NAMAGANDA, G. Testing the WISN method in Mbale and Mukono districts, Uganda, 2006. In: WORLD HEALTH ORGANIZATION. Applying the 
WISN method in practice: case studies from Indonesia, Mozambique and Uganda. Genebra: WHO, 2010. p.18-23.

NICARÁGUA. Ministério de Salud. Dirección Geral de Recursos Humanos. Manual de procedimientos de planificación y programación de recursos humanos. Managua: MINSA, 2007.

NICOLA, A. L. Dimensionamento do pessoal de enfermagem no Hospital Universitário do Oeste do Paraná. 2004. 153f. Tese (Doutorado em Enfermagem) - Escola de Enfermagem de Ribeirão Preto, Universidade de São Paulo, São Paulo, 2004.

NYANTEMA, A. S. et al. Staffing needs for quality perinatal care in Tanzania. African Journal of Reproductive Health, Benin City, v. 12, n. 3, p. 113-124, 2008.

\section{ORGANIZAÇÃO MUNDIAL DA SAÚDE (OMS).}

Trabalhando juntos pela saúde: Relatório Mundial de

Saúde 2006. Brasília: Ministério da Saúde, 2007.

PADILHA, K. G. et al. Nursing workload in intensive care units: a study using the Therapeutic Intervention Scoring System-28 (TISS-28). Intensive Critical Care Nursing, Edinburgh, v. 23, n. 3, p. 62-69, 2007.

PIERANTONI, C. R. et al. Carga de trabalho de um profissional típico da Atenção Primária em Saúde no Brasil: os agentes comunitários de saúde. Revista de Atenção Primária à Saúde, Juiz de Fora, v. 14, n. 4, p. 490-496, 2011a.

PIERANTONI, C. R. et al. Indicadores de carga de trabalho para profissionais da Estratégia Saúde da Família. In: PIERANTONI, C. R.; DAL POZ, M. R.; FRANÇA, T. (Org.). O Trabalho em Saúde: abordagens quantitativas e qualitativas. Rio de Janeiro: CEPESC: IMS/UERJ: ObservaRH, 2011b. p. 55-68.

PILLAY, T. et al. Neonatal nurse staffing and delivery of clinical care in the SSBC Newborn Network. Arch Dis Child Fetal Neonatal Ed., Londres, v. 97, p. 174-178, 2012.

POSSARI, J. F. Dimensionamento de profissionais de enfermagem em centro cirúrgico especializado em oncologia: análise dos indicadores intervenientes. 2011. 185f. Tese (Doutorado em Ciências) - Escola de Enfermagem, Universidade de São Paulo, São Paulo, 2011.

QUEIJO, A. F. Estudo comparativo de carga de trabalho de enfermagem em unidades de terapia intensiva geral $e$ especializadas segundo o Nursing Activities Score (NSA). 2008. 91f. Tese (Doutorado em Enfermagem) - Escola de Enfermagem, Universidade de São Paulo, São Paulo, 2008.

RABY, C. et al. Postpartum/newborn patients: who are they and do they all need the same amount of nursing care? J Nurs Man, Oxford, v. 16, n. 2, p. 198-203, 2008.

RAMOS, M. C. A.; CRUZ, L. P.; POLLARA, W. M. Desenvolvimento e aplicação de metodologia para o dimensionamento do corpo clínico em um hospital de ensino do município de São Paulo. Revista de Administração em Saúde, São Paulo, v. 14, n. 54, p. 3-10, 2012.

REAM, R. S. et al. Association of nursing workload and unplanned extubations in a pediatric intensive care unit. Pediatric Critical Care Medicine, Baltimore, v. 8, n. 4, p. 366-371, 2007.

REID, B.; KANE, K.; CURRAN, C. District nursing workforce planning: a review of the methods. British Journal of Community Nursing, Londres. v. 13, n. 11, p. 525-530. 2008.

REIS MIRANDA, D.; MORENO, R.; IAPICHINO, G. Nine equivalents of nursing manpower use score (NEMS). Revista Gaúcha de Enfermagem, Porto Alegre, v. 31, n. 3, p. 584-90, 2010.

ROSSETTI, A. C. Carga de trabalho de profissionais de enfermagem em pronto socorro: proposta metodológica. 2010. 116f. Dissertação (Mestrado em Enfermagem) -

Escola de Enfermagem, Universidade de São Paulo, São Paulo, 2010.

SANTOS, N. C. Construção de instrumento para identificação da carga de trabalho da equipe de enfermagem em unidades pediátricas. 2006. 109f. Dissertação 
(Mestrado em Enfermagem) - Escola de Enfermagem, Universidade de São Paulo, São Paulo, 2006.

SAWATZKY-DICKSON, D.; BODNARYK, K. Validation of a tool to measure neonatal nursing workload. Journal Nursing Management. Oxford, v. 17, n. 1, p. 8491, 2008.

SCHOO A. M. et al. Workload capacity measures of estimating allied health staffing requirements. Aust. Health Rev., Sidney. v. 10, n. 6, p. 376-82. 2008.

SERAN, S. et al. Decentralized application of the WISN method in Nusa Tenggara Timur Province, Indonesia, 2008. In: WORLD HEALTH ORGANIZATION. Applying the WISN method in practice: case studies from Indonesia, Mozambique and Uganda. Genebra: WHO, 2010. p. 1-9.

SOLIMAN, F. Improving resource utilization.through patient dependency systems. Journal of Medical Systems, New York, v. 21, n. 5, p. 291-302, 1997.

SPENCE, K. et al. Measuring nursing workload in neonatal intensive care. Journal of Nursing Management, Oxford, v. 14, n. 3, p. 227-234, 2006.

STENSKE, J.; FERGUSON, R. Analysis of labor and delivery workload. Nursing Management, Philadelphia, v. 27 , n. 6 , p. 30-32, 1996.

TSUKAMOTO, R. R. Tempo médio de cuidado ao paciente de alta dependência de enfermagem segundo o Nursing Activities Score (NAS). 2010. 107f. Dissertação (Mestrado em Enfermagem) - Escola de Enfermagem, Universidade de São Paulo, São Paulo, 2010.
TWIGG, D.; DUFFIELD, C. A review of workload measures: a context for a new staffing methodology in Western Australia. International Journal of Nursing Studies, Oxford, v. 46, n. 1, p. 131-139, 2009.

UPENIEKS, V. V. et al. Value-added care: a new way of assessing nursing staffing ratios and workload variability. Journal of Nursing Administration, Philadelphia. v. 37, n. 5, p. 243-52, 2007.

VOLPATTI, C. et al. Time-weighted nursing demand is a better predictor than midnight census of nursing supply in a intensive care unit. J. Critical Care. Philadelphia, v. 15, n. 4, p.147-150, 2000.

WALLIS, L. A.; TWOMEY, M. Workload and casemix in Cape Town emergency departments. South African Medical Journal, Cape Town, v. 97, n. 12, p. 1276-1280, 2007

WILLIS, E. et al. Calculating nurse staffing in community mental health and community health settings in South Australia. Nursing Forum, Hillsdale, v. 47, n. 1, p. $52-64,2012$.

WYSOKINSKI, M.; KSYKIEWICZ-DOROTA, A.; FIDECKI, W. Demand for nursing care for patients in intensive care units in South east Poland. American Journal of Critical Care, Aliso Viejo, v. 19, n. 2, p. 149-55, 2010.

\footnotetext{
Recebido para publicação em setembro de 2014 Versão final em dezembro de 2014

Conflito de interesses: inexistente

Suporte financeiro: CNPq. Processo 310404/2013-4 (Bolsa de Produtividade em Desenvolvimento Tecnológico e Extensão Inovadora) e Uerj, Programa de Incentivo à Produção Científica, Técnica e Artística (PROCIENCIA/Uerj/Faperj)
} 